\title{
Application of Low Temperature Plasma Technology in Crop Seeds
}

\author{
Bo Zhang* \\ Changzhou Vocational Institute of Mechatronic Technology, Changzhou, China \\ *Corresponding author: Bo Zhang, Changzhou Vocational Institute of Mechatronic Technology, Changzhou 213164, PR China
}

\begin{abstract}
Low-temperature plasma seed activation, as a new internationally developed agricultural technology, can stimulate seed vitality, improve seed quality, effectively enhance crop resistance to stress and growth, and increase crop yield. The innovative development of low-temperature plasma seed treatment technology is briefly introduced, the technical application of plasma technology in crop seeds, the biological effects of plasma and the activation mechanism are introduced, and the development and application prospects of plasma seed treatment technology are prospected. The low-temperature plasma crop seed treatment technology opens a new way for the application of plasma in crops.
\end{abstract}

Keywords: Low temperature Plasma; Seed; Activation; Equipment

\section{Introduction}

Low-temperature plasma is usually a kind of high-energy aggregated state produced by gas ionization through gas discharge. It contains many active particles such as electrons, ions, photons, excited molecular atoms, and free radicals. Plasma crop seed processing technology is the latest international research and development of new agricultural production [1-3]. The technology is the application of physical technology in the fields of biology and agriculture. The technology originated from aerospace science. Most of the crop seeds carried by the space launch showed abnormal growth vitality [4-7]. According to this revelation, the Russian National Institute of Physics first developed a plasma seed processing device to simulate the state of the ionosphere in space, using gas ions (plasma), rays, electromagnetic fields, vacuum and other factors to act on crop seeds and stimulate them. Its physiological activity and expression of potential stress resistance genes have improved crop vitality and resistance to drought and cold stress. The United States, Ukraine, South Korea, Israel and other countries also research and apply this technology. In addition, the United States, Canada and other countries are also researching lowtemperature plasma treatment to sterilize seeds for easy storage. In
China we took the lead in carrying out research on low-temperature plasma seed treatment technology, completed the entire process from equipment development, treatment dose determination, physiological mechanism discussion to demonstration application, and formed a practical series of plasma seed treatment crop yield increasing technology, which has achieved the following progress:

a. Developed low-temperature plasma crop seed processing equipment.

b. Through laboratory measurements of various physiological indicators of seed and seedling vigor, the relevant mechanism of low-temperature plasma seed processing was explored, and low-temperature plasma was confirmed from a physiological and metabolic perspective Seed treatment promotes the expression of stress resistance genes in crops, and provides a reliable theoretical basis for the application of plasma yield-increasing technology.

c. Through many indoor studies and field experiments, the general law of low-temperature plasma dose effects was found. The study determined 20 Suitable dosage range for seed treatment of various crops. 
d. Research and develop a series of practical techniques for increasing yield of plasma crops: wheat plasma drought resistance and yield increasing technology, corn plasma high yielding technology, soybean plasma increasing yielding technology, vegetable rapid seedling raising and efficient yield increasing technology and Plasma Aging Seeds complex technology.

e. Plasma seed treatment technology has been proven in production practice, a substantial increase crop yield.

\section{Low Temperature Plasma Seed Treatment Technol- ogy}

In recent years, Chinese agricultural researchers have also been exploring the low-temperature plasma seed treatment technology and have achieved practical application results [8-13]. The lowtemperature plasma seed processing technology is to place the seeds in the plasma and let the seeds contact the plasma, because the plasma is a high-energy aggregation state, which can penetrate the seed epidermis and interact with the seeds to cause some substances in the seeds to occur. Changes, thereby stimulating crop yields. To use plasma to treat seeds, we must first obtain a stable plasma generating device. The commonly used method is to ionize the air to form air plasma. Select an appropriate sealed container and evacuate the sealed container. The so-called vacuum is lower than the state of a gas at atmospheric pressure, one atmospheric pressure is tens of thousands of $\mathrm{Pa}$, and the vacuum pressure for generating plasma is generally about $100 \mathrm{~Pa}$. In this sealed container, two electrodes must also be installed, which can be composed of two conductive parallel plates. The structure is equivalent to a parallel plate capacitor. The electrode is connected to the outside by a wire. When the vacuum reaches about $100 \mathrm{~Pa}$, the power is turned on. Under a certain voltage, the air between the two parallel plate electrodes in the vacuum container is ionized. An air plasma is formed, and there are mainly nitrogen ions, oxygen ions, and electrons between the two electrode plates. Generally, in the plasma state, the glow discharge phenomenon is usually associated with this substance. This is the basic principle of the plasma generator. With the plasma generator, the seed of the crop is placed in a certain way between the two plates in the vacuum container, so that the plasma between the plates and the seeds contact each other. After a certain time and a certain intensity of plasma treatment, the activity of the seeds It can be improved, and the purpose of increasing crop yield can be achieved in production.

\section{Innovative Development of Low Temperature Plas- ma Seed Processing Technology}

The use of low-temperature plasma technology for seed treatment has become more and more recognized, and research and development of low-temperature plasma seed processing equipment has received increasing attention. Low-temperature plasma seed treatment technology has been commercialized in
Russia and has been popularized in production. In this regard, relevant domestic departments and units have also introduced some plasma modification treatment equipment from abroad, but also found that there are great shortcomings: the process repeatability of the equipment is very poor, and the energy consumption is high. Carefully study the situation of foreign equipment and found that the fundamental defect is that the discharge technology of the equipment is not well handled, that is, a serious DC discharge phenomenon has occurred in the radio frequency electric field. The root cause is due to the asymmetry of the electrodes, and the alternating current in the radio frequency In the electric field (13.56MHz), because the metal cylinder participates in one of the electrodes (ground) and discharges to the other electrode (target), a large amount of charge cannot be released and a DC potential is formed. As a result, the effective power of the RF electric field is very small and cannot be fully achieved Process repeatability requirements. There are also domestic technologies that use a radio frequency source to connect two parallel electrode plates to generate a glow discharge area. However, because a glow discharge can also occur between the two electrode plates and the inner wall of the cavity, resulting in a sharp increase in power consumption and waste of energy, In addition, a concentrated glow discharge region cannot be generated between the two plates, and a significant effect cannot be achieved. Low-temperature plasma seed processing equipment is a brand-new technology in China. Using Russian technology for reference, a plasma seed activation processing equipment has been developed, which has filled the domestic gap. The plasma seed activation processing equipment is mainly composed of three parts: a radio frequency matcher, a vacuum system, a plasma generating device and a transmission system. Main technical indicators: The volume of the working vacuum chamber is $\varphi 260 \mathrm{~mm} \times 1200 \mathrm{~mm}$, the gas pressure when generating plasma is $30 \mathrm{~Pa}-1 \times 105 \mathrm{~Pa}$, the working voltage is $380 \mathrm{~V}$, the frequency is $50 \mathrm{~Hz}$, the power is $50-1000 \mathrm{~W}$, and the discharge method is glow discharge.

\section{Biological Effects and Activation Mechanism of Low Temperature Plasma}

For the action mechanism of plasma seed treatment, a preliminary explanation can be made through experiments. Take a certain type of wheat as a comparative experiment, cultivate plasma treated wheat and untreated wheat in a petri dish, and cultivate under the same conditions. After one week, take one gram of germinated seedlings each, place them in a mortar, grind them into uniform slurry, put them into a graduated cylinder, dilute to a certain ratio, and then separate them with a centrifuge. Take the supernatant for use because various enzymes are dissolved in the supernatant, so using this supernatant can determine the activity of certain enzymes in wheat seedlings by certain technical means. The activities of the following enzymes were tested in the experiment: amylase (ATP), peroxidase (POD), superoxide dismutase (SOD), 
these three enzymes have a very important role in crop growth. Experiments show that these three enzymes are active after a certain amount of plasma treatment. Enzyme activity related to plasma treatment increased by $60.6-143.1 \%$, and ATP content increased by $9.1-62.2 \%$. Enzyme analysis showed that there was no change in the number of enzyme bands, but the enzyme activity was significantly enhanced. The ATP content of plant leaves increased by $13.9-178.5 \%$, and the root system increased by about $7.5 \%$. Under drought stress, the accumulation of osmolytes in crop leaves and roots increased, and the protective enzyme activities such as POD and SOD increased by 12.9-60.8\%. It reflects the promoting effect of plasma on seed germination and crops, and the drought resistance is significantly enhanced.

\section{Outlook}

Plasma is one of the current hotspots in physics research, and its development and application in the fields of biology and agriculture have just begun internationally. The mechanism of low temperature plasma seed treatment needs to be further studied, but it has increased crop yield and the effects shown by stress resistance and other aspects are obvious. Low-temperature plasma technology uses industrial production to promote agricultural production, which is a revolutionary new agricultural production technology. The application of plasma seed processing technology in agricultural production, one investment, long-term benefits, low operating costs, no variation, no pollution, significant yield increase effect, and strong practicability. For the vast number of farmers to accept this new agricultural technology, a process is needed. At the same time, more publicity efforts must be made. At the same time, there must be a certain amount of capital and technical investment. The equipment should be further improved and standardized as soon as possible to achieve batch and commercial production. Expanding the scale of application and creating greater economic and social benefits, the promotion of this technology will undoubtedly have a significant impact on the modernization of agricultural equipment.

\section{Acknowledgement}

This work has been supported by the Basic Research Fund for Jiangsu Science and Technology Support Program (No.
BK20161201) and Qing Lan Project of JiangSu Province (No. 2016). Helpful comments by Dr. Sao are appreciated.

\section{References}

1. Yekaterina Shapira, Victor Multanen, Gene Whyman, Yelena Bormashenko, Gilad Chaniel, et al. (2017) Plasma treatment switches the regime of wetting and floating of pepper seeds. Colloids and Surfaces B: Biointerfaces 157(9): 417-423.

2. Denis Butscher, Hanne Van Loon, Alexandra Waskow, Philipp Rudolf von Rohr, Markus Schuppler (2016) Plasma inactivation of microorganisms on sprout seeds in a dielectric barrier discharge. International Journal of Food Microbiology 238(12): 222-232.

3. Sang Hye Ji, Ki Hong Choi, Anchalee Pengkit, Jun Sup Im, Ju Sung Kim, et al. (2016) Effects of high voltage nanosecond pulsed plasma and micro DBD plasma on seed germination, growth development and physiological activities in spinach. Archives of Biochemistry and Biophysics 605(9): 117-128.

4. Khanit M (2016) Non-thermal Plasma for Germination Enhancement of Radish Seeds. Procedia Computer Science 86(2): 132-135.

5. Reoto O, Shohei U, Nobuya H, Rina Kosaka, Yasutaka Soeda (2017) Inactivation of bacteria on plant seed surface by low-pressure RF plasma using a vibrating stirring device. Vacuum 136(2): 214-220.

6. Daniela D, Monica M, Nicolae B, Maria D (2015) The effect of non-thermal plasma treatment on wheat germination and early growth. Innovative Food Science \& Emerging Technologies 29(5): 255-260.

7. ARM da Silva, ML Farias, DLS da Silva, JO Vitoriano, RC de Sousa, et al. (2017) Using atmospheric plasma to increase wettability, imbibition and germination of physically dormant seeds of Mimosa Caesalpiniafolia. Colloids and Surfaces B: Biointerfaces 157(9): 280-285.

8. Pradeep P, Je-Wook K, Chulkyoon M (2017) Effect of corona discharge plasma jet treatment on decontamination and sprouting of rapeseed (Brassica napus L.) seeds. Food Control 71(1): 376-382

9. Meiqiang Y, Mingjing H, Buzhou M, Tengcai M (2005) Stimulating Effects of Seed Treatment by Magnetized Plasma on Tomato Growth and Yield. Plasma Science \& Technology 7(6): 3143-3147.

10. Hajime S, Takehiro S, Ichiro T (2006) Expression analysis of genes encoding plasma membrane aquaporins during seed and fruit development in tomato. Plant Science 171(4): 277-285.

11. Marshal D, Sook Yong L, Sang-Un P (2006) Using low-pressure plasma for carthamus tinctorium seed surface modification. Vacuum 80(5): 499-506.

12. Bo Zhang, Ruihuan Li, Junchao Yan (2018) Study on activation and improvement of crop seeds by the application of plasma treating seeds equipment. Archives of Biochemistry and Biophysics 655(10): 37-42.

13. LI L, LI J, Shen M (2016) Improving Seed Germination and Peanut Yields by cold plasma treatment. Plasma Science \& Technology 18(10): 1027-1033. 
(c) (i) This work is licensed under Creative Commons Attribution 4.0 License To Submit Your Article Click Here: Submit Article

DOI: $10.32474 /$ CIACR.2020.08.000295

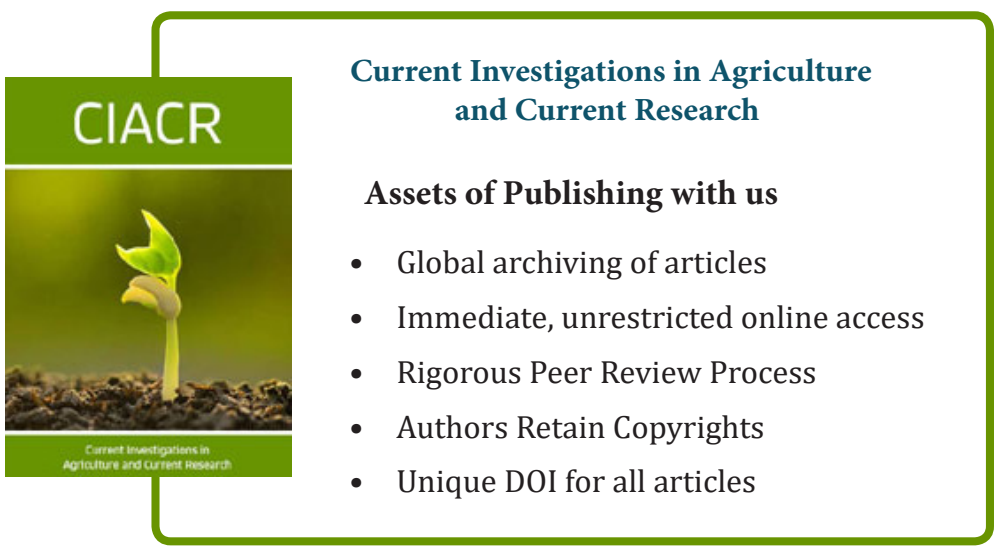

